\title{
Evaluación de la probabilidad de impacto de la Tierra con el Asteroide 2011 AG5
}

Roberto Schöngarth Carias

\section{Resumen}

El objeto NEA 2011AG5 es un asteroide de unos 140 metros de diámetro, descubierto el 8 de enero de 2011 desde el Monte Lemmon en Estados Unidos. Tiene una órbita que lo convierte en un asteroide potencialmente peligroso, o sea un objeto cuya órbita tiene una distancia menor a 0.05UA respecto a la órbita de la Tierra en su punto más cercano. Por sus características orbitales, se trata de un objeto tipo Apolo, o sea asteroides que cruzan la órbita de la Tierra con un perihelio menor a 1.0167UA. En este trabajo se modeló el movimiento orbital del objeto NEA 2011AG5 para un período aproximado de 28 años, que abarca desde el año 2012 hasta el año 2040 utilizando el integrador numérico EVORB, un programa basado en arquitectura FORTRAN para modelar la dinámica de los objetos en un sistema estelar. Se realizaron 100 corridas del programa con base en los parámetros orbitales estimados al 18 de abril de 2013. Después de asegurar que los parámetros orbitales de entrada, obtenidos aleatoriamente, cumplen con una distribución normal dado su Jarque Bera menor a 5.991 para un $\alpha=0.05$, se estimó que el máximo acercamiento ocurrirá el 4 de febrero de 2040. Para este acercamiento se estima una distancia media de 1,087,412 Km con una desviación estándar de 46,637 Km, en un acercamiento probable calculado para el 4 de febrero de 2040 a las 8h37m hora universal, con una desviación estándar de 48m04s. La probabilidad de impacto se concluye nula.

Palabras clave: 2011AG5, Asteroide cercano a la Tierra (NEA), Asteroide potencialmente peligrosos (PHA), acercamiento del 2040, asteroide Apolo.

\section{Abstract}

The NEA 2011AG5 object is an asteroid 140 meters in diameter, discovered on Jan. 8, 2011 from Mount Lemmon in the United States. It has an orbit that makes it a 
potentially hazardous asteroid, an object whose minimum distance to Earth's orbit is 0.05AU. Due to its orbital characteristics, it is an Apollo object, which means it is an asteroid that crosses the orbit of Earth having a perihelion smaller than 1.0167AU. The orbital motion of the object NEA 2011AG5 has been modeled for a period of approximately 28 years, from 2012 until 2040 using the numerical integrator EVORB, a program based on FORTRAN architecture to model the dynamics of objects in a stellar system. It has been developed 100 samples based on the orbital parameters estimated up to 18 April 2013. After ensuring that the random orbital parameters conform a normal distribution, due to its Jarque Bera is less than 5,991 for $\alpha=$ 0.05 , it was estimated that the closest approach will occur on February 4, 2040. An average distance of $1,087,412 \mathrm{~km}$ is estimated with a standard deviation of 46.637 $\mathrm{Km}$, in a calculated approach likely for February 4, 2040 at 8h37m universal time, with a standard deviation of $48 \mathrm{~m} 04 \mathrm{~s}$. The probability of impact is null.

Keywords: 2011AG5, Near Earth Asteroid (NEA), Potentially Hazardous Asteroids (PHA), close approach in 2040, Apollo asteroid.

Roberto Schöngarth Carías, (robertoschongarth@unah.edu.hn), Departamento de Astronomía y Astrofísica, Facultad de Ciencias Espaciales, Universidad Nacional Autónoma de Honduras - UNAH . 


\section{INTRODUCCIÓN}

Crear un modelo de la órbita de los cuerpos alrededor del Sol es parte del campo de la mecánica celeste. El presente trabajo trata de resolver este problema de mecánica fundamentado en el problema de los $\mathrm{N}$-Cuerpos, donde se describe el movimiento de un sistema de $n$ puntos $(n \geq 2)$ que se atraen dos a dos con una fuerza newtoniana (Navarro, Catalá Poch, \& Núñez de Murga, 2007). El movimiento de un planeta, cometa o asteroide alrededor del Sol, en primera aproximación, depende de seis elementos que definen la órbita. Al final esto constituye un sistema de $3 n$ ecuaciones diferenciales de segundo orden el cual debe resolverse utilizando métodos numéricos que han de ser resueltos mediante el uso de un software capaz de resolver integraciones numéricas de un sistema de ecuaciones diferenciales.

En el presente artículo se emplea un modelo orbital resuelto por uno de los programas informáticos para predicción de futuros impactos, en este caso el integrador numérico EVORB(Fernández, Gallardo, \& Brunini, 2002), basado en Fortran y, además, se propone una metodología de análisis estadístico para calcular peligros potenciales en función de una serie de escenarios generados aleatoriamente.

Este estudio es relevante en el sentido que día a día se están descubriendo nuevos objetos que tienen un paso cercano a la Tierra lo que los convierte en objetos potencialmente peligrosos para el planeta.

La incertidumbre $(1 \sigma)$ de los parámetros orbitales va disminuyendo a medida que aumenta la cantidad de observaciones del objeto. Utilizando un integrador numérico (EVORB) lo que se propone en el siguiente estudio es utilizar esta herramienta para estimar la probabilidad de impacto en el año 2040 utilizando datos obtenidos en dos épocas diferentes. Esto permitirá establecer si hay una consistencia entre los datos propios y los datos publicados en documentos científicos y así establecer el grado de precisión de la herramienta informática en uso. La familiarización y el uso de este integrador permitirán repetir la operación con futuros cuerpos y permitirá crear los modelos orbitales de otros objetos que pudieran acercarse peligrosamente a la Tierra.

El trabajo resultará en una utilidad metodológica en el sentido de poder aplicar localmente estas técnicas para el uso de integradores numéricos, no sólo para calcular probabilidades de impacto sino para poder estimar las posiciones y velocidades de objetos celestes para cualquier época. 


\section{El Sistema Solar}

El Sistema Solar tiene como objeto principal al Sol, el cual contiene más del $99.8 \%$ de la masa total del sistema. La masa de los cuerpos restantes no es en ningún sentido despreciable: los planetas, sus satélites, los cometas y el resto de cuerpos pequeños(Lissauer, 2013). Los planetas se dividen en dos grupos:

- Los planetas terrestres o interiores, que son rocosos, densos, con pocos satélites y sin anillos: Mercurio, Venus, Tierra y Marte.

- Los planetas jovianos o exteriores, que son gaseosos, poco densos, con muchos satélites y poseedores de sistemas de anillos: Júpiter, Saturno, Urano y Neptuno.

De igual manera se encuentran dos cinturones que poseen cada uno cientos de miles de cuerpos pequeños:

- El cinturón de asteroides, formado por cuerpos pequeños, irregulares y rocosos, los cuales en su gran mayoría orbitan entre Marte y Júpiter. Algunos de ellos poseen órbitas interiores al planeta Marte e incluso se acercan lo suficiente a la Tierra para ser considerados potencialmente peligrosos.

- El cinturón de Kuiper, compuesto por cuerpos pequeños y helados que orbitan al Sol en una órbita más alejada que Neptuno. El primero de ellos, 1992QB1 fue descubierto en 1992. Este cinturón se extiende entre 30 y 48UA de distancia respecto al Sol.

Más allá del Cinturón de Kuiper se están descubriendo nuevos objetos transneptunianos, habiéndose observado de forma directa objetos a más de 500UA. El Sistema Solar se extiende hasta lo que se conoce como Nube de Oort, una hipotética nube de objetos rocosos helados, mitad silicatos y mitad volátiles, cuya distancia se estima hasta aproximadamente varias decenas de miles de unidades astronómicas.

En el medio interplanetario del Sistema Solar también se encuentran objetos conocidos como meteoroides. Si un meteoroide penetra la atmósfera terrestre comienza a desintegrarse debido a la fricción, por lo cual se observa una estela de luz en el cielo conocida como meteoro o estrella fugaz. Si este meteoroide tiene un tamaño o masa suficiente como para sobrevivir la interacción con la atmósfera y llega a impactar la superficie de la Tierra, entonces se le conoce como meteorito(Rubin \& Grossman, 2010). El tamaño de un meteoroide está entre los $10 \mu \mathrm{m}$ y un metro, de los cuales los que tienen menos de $2 \mathrm{~mm}$ de diámetro son 
considerados micrometeoroides. Objetos menores a 10um son considerados partículas de polvo. La frontera de tamaño entre los meteoroides y los asteroides se ha asumido en función de su capacidad de detección, donde ya un cuerpo de 1 metro de diámetro es mucho más fácil de localizar. En este caso de cuerpos mayores a un metro de tamaño, se habla de asteroides, los cuales son el objetivo central del presente estudio.

Un asteroide es denominado NEA (Asteroide cercano a la Tierra, del inglés Near Earth Asteroid) cuando su trayectoria lo lleva a menos de 1.3UA del Sol (1 unidad astronómica $=1 \mathrm{UA}=149,587,870,700 \pm 3 \mathrm{~m}$, según la resolución B2 de la XXVIII Asamblea General de la Unión Internación de Astronomía), de los cuales el mayor conocido hasta ahora es 1036 Ganymed, de $31.7 \mathrm{Km}$ de diámetro y un período alrededor del Sol de 4.34 años(Chodas, Near Earth Object Program, 2016).

Más cercanos aparecen los PHA (Asteroides potencialmente peligrosos, del inglés Potencially Hazardous Asteroids) pues su acercamiento máximo a la Tierra llega a ser menor a las 0.05UA, equivalentes a casi 20 distancias lunares. El mayor conocido es 4179 Toutatis, un objeto de $4.6 \times 2.4 \times 1.9 \mathrm{Km}$ y con un período orbital de 4.03 años.

Hasta octubre del 2016 se han descubierto más de 15,000 objetos NEA (20 años atrás la cantidad apenas sobrepasaba los 300 ) aunque se estima que la cantidad real de los mismos sea de varios cientos de miles. En la Figura 1(Chodas, Near Earth Object Program, 2016)se observa el ritmo del descubrimiento de NEAs de diámetro mayor a un kilómetro por cada semestre desde 1995. En el eje horizontal se observan los años de los descubrimientos y en el eje vertical la cantidad de descubrimientos por semestre.

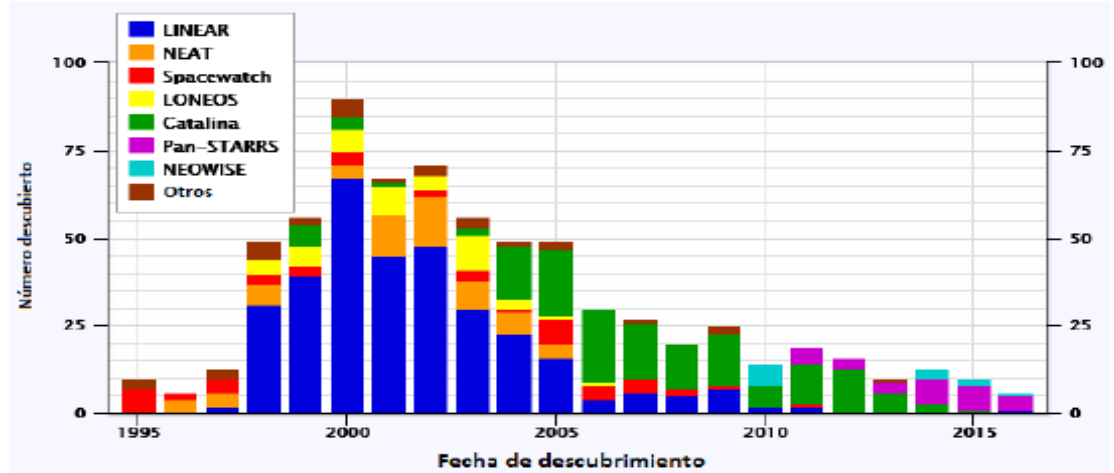

Figura 1. Número de NEAs de diámetro mayor a $1 \mathrm{Km}$ descubiertos por sitio en intervalos de medio año(Chodas, Near Earth Object Program, 2016) 
Cada barra correspondiente a un semestre ha sido coloreada en función de la cantidad de descubrimientos por cada uno de los proyectos de búsqueda de NEAs, razón por la cual resaltan los descubrimientos por parte del proyecto LINEAR (azul), Catalina (verde) y Pan-STARRS (morado). Al observar la curva de descubrimiento de NEAs de tamaño grande (diámetro mayor a un kilómetro) se observa en la Figura 1 que la curva de descubrimiento se ha ido reduciendo de manera sostenida durante los últimos 10 años. Hasta octubre de 2016 se han descubierto 706 asteroides de más de un kilómetro de tamaño.

\section{Clasificación de los NEAs Según Sus Órbitas}

En la base de datos al 31 de octubre de 2016 se contabilizan 15277 NEOs (Near Earth Objects, objetos cercanos a la Tierra), que corresponden a la suma de los 15103 NEAs (Near Earth Asteroids, asteroides cercanos a la Tierra) con los 174 NECs (Near Earth Comets, cometas cercanos a la Tierra)(Chodas, Near Earth Object Program, 2016).

Los NEAS se clasifican según la distancia al Sol, sea distancia media, sea la distancia del perihelio, en cuatro importantes categorías (ver Figura 2):

\begin{tabular}{|l|l|}
\hline Asteroide Apolo & $\begin{array}{l}\text { Semieje mayor } \geq 1.0 \mathrm{UA} \\
\text { Perihelio } \leq 1.02 \mathrm{UA} \\
\text { Cruza órbita de la Tierra }\end{array}$ \\
\hline Asteroide Atón & $\begin{array}{l}\text { Semieje mayor <1.0 UA } \\
\text { Afelio } \geq 0.983 \mathrm{UA} \\
\text { Cruza órbita de la Tierra }\end{array}$ \\
\hline Asteroide Amor & $\begin{array}{l}1.02 \mathrm{UA}<\text { Perihelio } \leq 1.3 \mathrm{UA} \\
\text { Asteroide Atira }\end{array}$ \\
$\begin{array}{l}\text { Afelio }<0.983 \mathrm{UA} \\
\text { Siempre dentro de la órbita terrestre }\end{array}$
\end{tabular}

Figura 2. Categorías de Near Earth Asteroids(Chodas, Near Earth Object Program, 2016) 
- Los asteroides Atones, que tienen un semieje menor que es inferior a una unidad astronómica pero, por el contrario, su afelio debe ser mayor a 0.9833 unidades astronómicas (este número corresponde al perihelio terrestre),

- Los asteroides Apolo, que tienen un semieje mayor que es superior a una unidad astronómica, pero su perihelio es menor a 1.0167 unidades astronómicas (este número corresponde al afelio terrestre)

- Los asteroides Amor, que se caracterizan por tener un perihelio no menor a 1.0167UA (o sea mayor al afelio terrestre) y no mayor a 1.3UA (o sea menor al perihelio de Marte).

- Los asteroides Atira, que incluyen todos los asteroides cuyo afelio es menor a $0.9833 \mathrm{UA}$, por lo tanto menor al perihelio terrestre.

Nótese que de estas categorías, los asteroides Apolo y Atón son los que se cruzan con la órbita terrestre, razón por la cual representan un mayor peligro. Se ha creado una categoría de Asteroides Potencialmente Peligrosos (PHA, Potencially Hazardous Asteroids), cuyas probabilidades de impacto con la Tierra son mayores. Para ello deben cumplir con dos requisitos(Chodas, Near Earth Object Program, 2016):

- Los puntos más cercanos entre la órbita del asteroide y la órbita terrestre se encuentran a menos de 0.05UA (aproximadamente 7.5 millones de kilómetros).

- La magnitud absoluta del asteroide debe ser de H=22.0 o más brillante.

Un $18 \%$ de asteroides Apolo y un $14 \%$ de asteroides Atón, aproximadamente, están dentro de los PHAs.

La Tabla 1 resume la cantidad de NEAs descubiertos, según cada categoría, hasta octubre de 2016. Las categorías se muestran en las columnas y corresponden a los asteroides Amor (AMO), Apolo (APO), Atón (ATE) y Atira (IEO). Además se puede observar cuántos asteroides de cada categoría son potencialmente peligrosos, según la definición antes descrita, para el planeta Tierra.

Tabla 1. Cantidad de NEAs y su peligrosidad según la categoría

\begin{tabular}{|c|c|c|c|c|c|c|}
\hline & & & Clas & ase & & \\
\hline & & AMO & APO & ATE & IEO & Total \\
\hline Asteroide notencialmente peligroso & No & 5759 & 6639 & 948 & 11 & 13357 \\
\hline 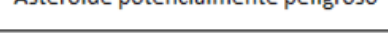 & Si & 108 & 1475 & 158 & 5 & 1746 \\
\hline Total & & 5867 & 8114 & 1106 & 16 & 15103 \\
\hline
\end{tabular}


El objeto 2011AG5 fue descubierto el 8 de enero de 2011 desde la cima del Monte Lemmon, en Arizona y a partir de entonces continuas observaciones han permitido ir ajustando su órbita y tratando de predecir sus pasos cercanos, en particular los que se darán entre los años 2040 y 2047.

Este estudio permitirá revelar la manera en cómo ajustar los datos correctamente ante los pasos de este objeto cerca de la Tierra. Estos cálculos no sólo serán útiles para el estudio de 2011AG5 en particular sino que para múltiples acercamientos de otros objetos potencialmente más peligrosos, como por ejemplo cuando el asteroide Apophis tenga un acercamiento similar en el año 2029, sólo que en ese caso es un objeto de un diámetro 6 veces mayor.

Al revisar los elementos orbitales del NEA 2011AG5, éste cumple con tres características que permiten incluirlo en cierta categoría de NEAs:

- El semieje mayor de su órbita es de 1.43UA, o sea que es exterior a la órbita de la Tierra.

- El perihelio de su órbita es de 0.87UA; al ser el semieje mayor exterior a la órbita terrestre y el perihelio menor al afelio terrestre, se concluye que este asteroide cruza la órbita terrestre. Combinando ambas características resulta que este asteroide pertenece a la categoría de los asteroides Apolo.

- Las órbitas de 2011AG5 y la de la Tierra se acercan a una distancia menor a las 0.05UA (7.5 millones de kilómetros), y el asteroide 2011AG5 tiene una magnitud absoluta menor a 22, lo que permite clasificar a este asteroide como un Apolo potencialmente peligroso.

Las observaciones han llevado a los siguientes hallazgos acerca de 2011AG5(Chodas, Sentry Risk Table, 2016):

- $\quad$ Diámetro $=140$ metros

- $\quad$ Magnitud absoluta $\mathrm{H}=21.86$

- $\quad$ Período $=625.1$ días

Se esperan acercamientos en cada órbita, pero los mayores acercamientos se esperan para los años 2023, 2028 y 2040. El del 2023 será todavía a aproximadamente millón y medio de kilómetros, pero se espera su máximo acercamiento en el año de 2040. 
Durante el año 2040 se prevé que se tendrá el primer acercamiento de 2011AG5 a una posible distancia menor al millón de kilómetros, a menos de tres distancias Tierra-Luna. Para este tipo de objetos y acercamientos potenciales se desarrolló la Escala de Palermo(Chodas, The Palermo Technical Impact Hazard Scale, 2016).

La escala de Palermo acerca de los riesgos de impacto fue desarrollada por especialistas en NEOs para clasificar posibles riesgos de impacto basados en fechas, energías y probabilidades. Se trata de una escala logarítmica donde el cero señala un evento que es tan amenazador como el peligro de fondo, que no es más que el peligro promedio generado por la población completa de asteroides y cometas sobre un largo período de tiempo. Valores superiores al cero corresponderán a eventos muy amenazadores, aunque valores entre 0 y -2 (una amenaza del 1\% respecto al peligro de fondo) comprende un riesgo aún no despreciable. En el momento en que se estimó el máximo riesgo del objeto 2011AG5, éste llegó a tener un índice de -1.12 en la Escala de Palermo. El 21 de diciembre de 2012 fue sacado de la lista de objetos con riesgo de impacto(Chodas, Near Earth Object Program, 2016).

La órbita de un objeto puede describirse a través de seis parámetros o elementos orbitales:

- La longitud del nodo ascendente $\Omega$, que mide la longitud donde la órbita del objeto cruza de manera ascendente, medida sobre la eclíptica a partir del punto Aries.

- La inclinación i es la inclinación de la órbita del objeto respecto al plano de la eclíptica.

- La longitud del perihelio $\omega$, que mide el ángulo visto desde el sol, desde el punto del nodo ascendente respecto a la dirección donde se da el perihelio.

- El semieje mayor a de la elipse descrita por la órbita del objeto.

- La excentricidad e que indica qué tanto se asemeja la elipse a un círculo.

- Tiempo de paso por el perihelio t, o sea el último paso del objeto por el perihelio. 
Los elementos orbitales de 2011AG5, junto con una incertidumbre de $1 \sigma$, para la época 2456400.5 (18 de abril 2013), son los siguientes(Park, 2015):

- Excentricidad: $0.390200698625485 \pm 7.1842 \mathrm{e}-08$

- Semieje mayor: $1.430761044178588 \pm 1.0983 \mathrm{e}-07 \mathrm{UA}$

- Inclinación de la órbita: $3.680379426804628 \pm 1.0164$ e-05 grados

- Nodo ascendente: $135.6848051202182 \pm 6.507$ e-05 grados

- Longitud del perihelio: $53.53870886708997 \pm 6.597$ e-05 grados

- Paso por el perihelio: 2012-Dic-08.38530278 \pm 7.0025 e-05 días

\section{METODOLOGÍA}

Al ser operaciones matemáticas de cierta complejidad, pues implican cálculo diferencial, se hace uso de software astronómico orientado a la mecánica celeste. Este consiste básicamente de un programa escrito en lenguaje FORTRAN de integraciónnumérica de las orbitas del Sistema Solar denominado EVORB (Fernández, Gallardo, \& Brunini, 2002). Con este software es posible hacer diversas simulaciones a largo plazo, tanto moviéndose hacia adelante en el tiempo como hacia atrás, para poder estimar tanto futuros acercamientos del objeto como posibles regiones de proveniencia.

Con los resultados obtenidos del modelo se hacen estimaciones estadísticas acerca de la probabilidad tanto de hacia dónde se espera que vaya y eventualmente cuándo y cómo serán futuros acercamientos a nuestro planeta en el próximo siglo.

La tabla 2 contiene los parámetros orbitales y muestra el valor medio más probable así como la desviación estándar. Basado en ello se procede a crear los escenarios para el cálculo de las probabilidades de impacto para el año 2040.En este caso se trabaja con los parámetros orbitales de semieje mayor, excentricidad, inclinación de la órbita, nodo ascendente, longitud del perihelio y anomalía media. 
Tabla 2. Elementos orbitales de 2011 AG5 (Park, 2015)

\begin{tabular}{|l|r|r|l|}
\hline \multicolumn{4}{|c|}{ Elementos orbitales en la época 2456400.5 (2013-Abril-18.0) } \\
\hline Elemento & \multicolumn{1}{|c|}{ Valor } & Incertidumbre (1 sigma) & Unidades \\
\hline Excentricidad & 0.390200698625485 & $7.1842 \mathrm{e}-08$ & \\
\hline Semieje mayor & 1.430761044178580 & $1.0983 \mathrm{e}-07$ & UA \\
\hline Perihelio & 0.872477085173974 & $6.018 \mathrm{e}-08$ & UA \\
\hline Inclinación de la órbita & 3.680379426804620 & $1.0164 \mathrm{e}-05$ & grados \\
\hline Nodo ascendente & 135.684805120218000 & $6.507 \mathrm{e}-05$ & grados \\
\hline Argumento del perihelio & 53.538708867089900 & $6.5975 \mathrm{e}-05$ & grados \\
\hline Anomalia media & 75.222101365178700 & $4.8726 \mathrm{e}-05$ & grados \\
\hline Tiempo de paso por el perihelio & $2012-D i c-08.38530278$ & $7.0025 \mathrm{e}-05$ & dias \\
\hline Periodo & 625.099407560966000 & $7.1976 \mathrm{e}-05$ & dias \\
\hline Movimiento verdadero & 0.575908400560896 & $6.6312 \mathrm{e}-08$ & grados/día \\
\hline Afelio & 1.989045003183200 & $1.5268 \mathrm{e}-07$ & UA \\
\hline
\end{tabular}

La Tabla 2 muestra los valores medios y desviaciones estándar de los parámetros orbitales de 2011AG5. Basado en ello, se procede a una generación por computadora de 100 escenarios aleatorios modelados por una distribución normal, para asegurar que al final los valores de media y desviación estándar de cada parámetro se asemejen a los valores observados. Cuando ya los escenarios se han generado, se procede a utilizar el programa EVORB, que consiste en un programa que fue desarrollado originalmente por Adrián Brunini (Argentina) y perfeccionado posteriormente por Tabaré Gallardo (Uruguay).

El EVORB determina la evolución de los elementos orbitales heliocéntricos (semieje mayor, excentricidad, inclinación de la órbita, longitud de nodo ascendente, argumento del perihelio y anomalía media) de un sistema de planetas que son descritos con su masa y su radio, así como un sistema de partículas (los cuales podemos considerar asteroides y cometas), los cuales son asumidos sin radio ni masa.

Estos planetas y partículas están bajo el efecto gravitacional de un cuerpo central, en este caso el Sol, aunque puede ser utilizado también en cálculos para otras estrellas. Estos objetos describen órbitas alrededor de este cuerpo central.

El EVORB considera la interacción gravitacional entre los planetas, así como el efecto de los planetas sobre las partículas sin masa, las cuales no interactúan entre ellas ni afectan el movimiento de los planetas. 
La salida del programa EVORB muestra una serie de resultados que pueden ser sometidos a análisis. En función de la interacción del cuerpo de estudio, en este caso 2011AG5, se muestra la posición y velocidad para cada intervalo de la iteración tanto del asteroide como de la Tierra. Esto permite obtener dos datos importantes:la distancia a la que se encuentran los cuerpos en el momento del máximo acercamiento y la velocidad individual y relativa de los mismos.

Esto es importante porque la velocidad relativa debe ser comparada con la velocidad de escape para saber si el asteroide puede ser atraído por la aceleración gravitacional de la Tierra. Sería incompleto en este estudio solamente suponer y estimar la probabilidad de un impacto directo entre ambos cuerpos sino que se debe incluir la posibilidad que el asteroide se acerque lo suficiente a la Tierra como para que comience a ser atraído por ésta y finalmente impactar. Esto se logra a través del estudio de la velocidad de escape.

La velocidad de escape es la velocidad requerida para escapar de la superficie de un cuerpo astronómico.

La velocidad de escape $V_{e}$, está dada por una ecuación bastante simple (Seeds, 2008):

$$
V_{e}=\sqrt{\frac{2 G M}{r}}
$$

donde en este caso M corresponde a la masa de la Tierra, o sea $5.97 \times 10^{24} \mathrm{Kg}$ y $\mathrm{G}$ es la constante gravitacional, que es igual a $6.67 \times 10^{-11} \mathrm{~m}^{3} / \mathrm{s}^{2} \mathrm{Kg}$ yr corresponde al radio de la órbita.

\section{ANÁLISIS DE RESULTADOS}

Tabla 3. Valores estadísticos medios de los parámetros orbitales de los cien escenarios generados

\begin{tabular}{|l|r|r|r|r|r|r|}
\hline Escenario & \multicolumn{1}{|c|}{$\mathrm{a}$} & \multicolumn{1}{c|}{$\mathrm{e}$} & \multicolumn{1}{c|}{$\mathrm{i}$} & \multicolumn{1}{c|}{ Nodo } & \multicolumn{1}{c|}{ Peri } & \multicolumn{1}{c|}{$\mathrm{M}$} \\
\hline Media & 1.430761 & 0.390201 & 3.680380 & 135.684811 & 53.538713 & 75.222094 \\
\hline Desviación estándar & $9.7101 \mathrm{E}-08$ & $6.5105 \mathrm{E}-08$ & $1.0334 \mathrm{E}-05$ & $6.1593 \mathrm{E}-05$ & $6.4649 \mathrm{E}-05$ & $4.8409 \mathrm{E}-05$ \\
\hline Asimetría & 0.026066 & 0.020617 & -0.106113 & -0.176965 & -0.153566 & -0.221927 \\
\hline Curtosis & -0.567331 & -0.685264 & -0.481491 & -0.280177 & 0.228732 & -0.240649 \\
\hline Jarque Bera & 1.352425 & 1.963694 & 1.153641 & 0.849024 & 0.611036 & 1.062157 \\
\hline
\end{tabular}


En la Tabla 3 se presenta un resumen de la estadística descriptiva de los parámetros orbitales que fueron utilizados en las cien corridas de datos. La razón para esta tabla resumen es precisamente observar que los escenarios propuestos se adecúan a los valores de media y desviación estándar que son comparables a los datos reales.

La asimetría y la curtosis muestran las medidas de forma que indican si los cien datos que se utilizaron para los cálculos se adecúan a una distribución normal. La asimetría y la curtosis deben ser cercanas a cero para tener la forma de una distribución normal.

El Jarque Bera consiste en una prueba de normalidad, precisamente para verificar que los escenarios aleatorios precisamente cumplen con la media y la desviación estándar de los parámetros orbitales observados. La prueba Jarque Bera se corre con un chi-cuadrado de dos grados de libertad, lo que para un $a=0.05$ resulta en un valor crítico de 5.991. Esto quiere decir que si el valor del Jarque Bera es menor a 5.991, la evidencia indica que se está trabajando con distribuciones normales.

En este caso, los valores de Jarque Bera oscilan entre 0.61 y 1.96 , siendo el valor para la excentricidad el más elevado aunque lejano aún a 5.991. Con esto se confirma que todos los parámetros orbitales describen una forma normal, con los valores de media y desviación estándar deseados, para los 100 datos con los que se trabaja y se puede proceder a correr las iteraciones.

A partir de los resultados de las iteraciones se puede afirmar lo siguiente:

El máximo acercamiento en el año 2040 entre la Tierra y 2011 AG5 se dará el 4 de febrero de 2040 a las 8:37:24 am con una desviación estándar aproximada de 48 minutos. Este acercamiento se dará a una distancia esperada de 1,087,412 kilómetros, con una desviación estándar de 46,637 kilómetros.

Tabla 4. Prueba t para la probabilidad de impacto de 2011 AG5 contra la Tierra en 2040

\begin{tabular}{|c|c|c|c|c|c|c|c|c|c|}
\hline & \multirow[b]{3}{*}{ N } & \multirow[b]{3}{*}{ Media } & \multirow{3}{*}{$\begin{array}{c}\text { Desviación } \\
\text { estándar }\end{array}$} & \multicolumn{6}{|c|}{ Valor de prueba $=6378$} \\
\hline & & & & \multirow[b]{2}{*}{ t } & \multirow[b]{2}{*}{ gl } & \multirow[b]{2}{*}{ Sig. (bilateral) } & \multirow{2}{*}{$\begin{array}{l}\text { Diferencia de } \\
\text { medias }\end{array}$} & \multicolumn{2}{|c|}{$\begin{array}{c}\text { 95\% de intervalo de confianza } \\
\text { de la diferencia }\end{array}$} \\
\hline & & & & & & & & Inferior & Superior \\
\hline distancia & 100 & 1087412,671 & 46637.74916 & 231.794 & 99 & .000 & 1081034,671 & 1071780,730 & 1090288,612 \\
\hline
\end{tabular}

La Tabla 4 muestra un resumen de los datos recabados en las cien pruebas realizadas. Los datos válidos para el análisis son $\mathrm{N}=100$, para los cuales se 
obtuvo una distanciamedia de 1,087,412.67 Km con una desviación estándar de $46637.75 \mathrm{Km}$.

En base a estos valores se establece una prueba de hipótesis que se plantea de la forma siguiente:

$\mathrm{H}_{0}$ : la distancia en el máximo acercamiento no es mayor a $6378 \mathrm{Km}$

$\mathrm{H}_{1}$ : la distancia en el máximo acercamiento es mayor a $6378 \mathrm{Km}$

Estadísticamente sería más correcto plantear una distancia estadística entre -6378 y $6378 \mathrm{Km}$, pero al observar los resultados se verá que esto no será necesario. Continuando con los valores de la tabla se observa que el valor de t es de 231.8 aproximadamente. Solamente al observar este valor se asegura que la hipótesis nula se rechaza, lo que implica que la distancia a la que pasará el asteroide 2011 AG5 será mayor al radio ecuatorial de la Tierra. En realidad el resultado es abrumadoramente superior. Estadísticamente valores de $t$ superiores a tres ya implican un moderado grado de seguridad de rechazo de la hipótesis nula, así que un valor de 231.8 no deja margen de duda.

Esto es apoyado por la significancia bilateral, que con tres valores decimales muestra que esta significancia es de $p=0.000$. Cabe aclarar que la hipótesis de investigación está planteada de la forma "mayor a" lo que representa una prueba estadística de una cola, por lo que la significancia estimada es la significancia bilateral dividida entre dos. Esto no afecta el resultado pues $p=0.000 / 2=0.000$.

Esto implica que la probabilidad de hallar un valor de prueba menor a $6378 \mathrm{Km}$ es nula, lo cual ya se demostró en la sección precedente del análisis de resultados.

Por lo tanto, utilizando las pruebas estadísticas mostradas en esta sección, no cabe duda que la probabilidad de impacto entre la Tierra y el asteroide 2011 AG5 puede asumirse como nula.

La Tabla 5 muestra la estadística descriptiva de la distancia de los acercamientos en el 2040 entre la Tierra y 2011AG5, donde se muestra una media de 1,087,412.7 kilómetros con una desviación estándar de 46637.7 km.

La proyección de la media de la población con un intervalo de confianza del 95\% indica que existe una certeza del $95 \%$ que el valor verdadero de la media se encuentra entre 1,078,158.7 y 1,096,666.6 kilómetros. 
Tabla 5. Estadísticas descriptivas de la distancia mínima de acercamiento para cada una de las muestras

\begin{tabular}{|c|c|c|c|c|}
\hline & & & Estadístico & $\begin{array}{c}\text { Error } \\
\text { estándar }\end{array}$ \\
\hline \multirow[t]{13}{*}{ Distancia mínima } & \multicolumn{2}{|l|}{ Media } & 1087412.6710 & 4663.77492 \\
\hline & \multirow{2}{*}{$\begin{array}{l}95 \% \text { de intervalo de } \\
\text { confianza para la media }\end{array}$} & Limite inferior & 1078158.7298 & \\
\hline & & $\begin{array}{l}\text { Límite } \\
\text { superior }\end{array}$ & 1096666.6122 & \\
\hline & \multicolumn{2}{|l|}{ Media recortada al $5 \%$} & 1087018.3852 & \\
\hline & \multicolumn{2}{|l|}{ Mediana } & 1087138.6950 & \\
\hline & \multicolumn{2}{|l|}{ Varianza } & 2175079646.585 & \\
\hline & \multicolumn{2}{|l|}{ Desviación estándar } & 46637.74916 & \\
\hline & \multicolumn{2}{|l|}{ Mínimo } & 987720.54 & \\
\hline & \multicolumn{2}{|l|}{ Máximo } & 1211128.96 & \\
\hline & \multicolumn{2}{|l|}{ Rango } & 223408.42 & \\
\hline & \multicolumn{2}{|l|}{ Rango intercuartil } & 61183.58 & \\
\hline & \multicolumn{2}{|l|}{ Asimetria } & .163 & 241 \\
\hline & \multicolumn{2}{|l|}{ Curtosis } & -.261 & 478 \\
\hline
\end{tabular}

La tabla 6 indica las fechas, las horas y las distancias de mayor acercamiento de los 100 escenarios propuestos. El acercamiento a menor distancia se da en el escenario 45, a apenas $987,720.54$ kilómetros. El acercamiento a mayor distancia se da en el escenario 44, a 1,211,128.96 kilómetros.

Tabla 6. Estimación de la fecha, hora y distancia de los máximos acercamientos Tierra-2001AG5 para cada una de las 100 corridas realizadas

\begin{tabular}{|ccccc|}
\hline Escenario & Fecha & Hora & Km & Vrel $(\mathrm{Km} / \mathrm{seg})$ \\
1 & $04 / 02 / 2040$ & $09: 00: 00$ a.m. & 1070451.891 & 9.7832 \\
2 & $04 / 02 / 2040$ & 10:00:00 a.m. & 1022501.68 & 9.7756 \\
3 & $04 / 02 / 2040$ & $08: 35: 00$ a.m. & 1096443.659 & 9.7873 \\
4 & $04 / 02 / 2040$ & $09: 00: 00$ a.m. & 1060088.014 & 9.7816 \\
5 & $04 / 02 / 2040$ & $08: 25: 00$ a.m. & 1094234.222 & 9.7870 \\
6 & $04 / 02 / 2040$ & $09: 05: 00$ a.m. & 1071001.995 & 9.7832 \\
7 & $04 / 02 / 2040$ & $09: 00: 00$ a.m. & 1058871.994 & 9.7814 \\
\hline
\end{tabular}




\begin{tabular}{|c|c|c|c|c|}
\hline 8 & $04 / 02 / 2040$ & 09:00:00 a.m. & 1076168.119 & 9.7841 \\
\hline 9 & $04 / 02 / 2040$ & 07:55:00 a.m. & 1129714.743 & 9.7928 \\
\hline 10 & $04 / 02 / 2040$ & 08:35:00 a.m. & 1098068.53 & 9.7876 \\
\hline 11 & $04 / 02 / 2040$ & 09:10:00 a.m. & 1058157.102 & 9.7813 \\
\hline 12 & $04 / 02 / 2040$ & 08:50:00 a.m. & 1081073.757 & 9.7849 \\
\hline 13 & $04 / 02 / 2040$ & 08:15:00 a.m. & 1105315.739 & 9.7889 \\
\hline 14 & $04 / 02 / 2040$ & 07:20:00 a.m. & 1152550.619 & 9.7966 \\
\hline 15 & $04 / 02 / 2040$ & 08:35:00 a.m. & 1093658.015 & 9.7869 \\
\hline 16 & $04 / 02 / 2040$ & 09:00:00 a.m. & 1066389.151 & 9.7826 \\
\hline 17 & $04 / 02 / 2040$ & 07:50:00 a.m. & 1125395.553 & 9.7921 \\
\hline 18 & $04 / 02 / 2040$ & 08:50:00 a.m. & 1079850.448 & 9.7847 \\
\hline 19 & $04 / 02 / 2040$ & 09:30:00 a.m. & 1045421.635 & 9.7792 \\
\hline 20 & $04 / 02 / 2040$ & 08:25:00 a.m. & 1107387.424 & 9.7891 \\
\hline 21 & $04 / 02 / 2040$ & 09:00:00 a.m. & 1081955.936 & 9.7850 \\
\hline 22 & $04 / 02 / 2040$ & 09:10:00 a.m. & 1062020.339 & 9.7819 \\
\hline 23 & $04 / 02 / 2040$ & 08:25:00 a.m. & 1104944.488 & 9.7887 \\
\hline 24 & $04 / 02 / 2040$ & 09:00:00 a.m. & 1056712.974 & 9.7811 \\
\hline 25 & $04 / 02 / 2040$ & 10:25:00 a.m. & 992833.1805 & 9.7709 \\
\hline 26 & $04 / 02 / 2040$ & 08:05:00 a.m. & 1108142.333 & 9.7893 \\
\hline 27 & $04 / 02 / 2040$ & 10:00:00 a.m. & 997275.2723 & 9.7717 \\
\hline 28 & $04 / 02 / 2040$ & 09:10:00 a.m. & 1057612.628 & 9.7811 \\
\hline 29 & $04 / 02 / 2040$ & 07:55:00 a.m. & 1138549.679 & 9.7942 \\
\hline 30 & $04 / 02 / 2040$ & 09:40:00 a.m. & 1014299.143 & 9.7743 \\
\hline 31 & $04 / 02 / 2040$ & 09:00:00 a.m. & 1060771.255 & 9.7817 \\
\hline 32 & $04 / 02 / 2040$ & 07:15:00 a.m. & 1175075.593 & 9.8003 \\
\hline 33 & $04 / 02 / 2040$ & 08:35:00 a.m. & 1095353.686 & 9.7872 \\
\hline 34 & $04 / 02 / 2040$ & 08:50:00 a.m. & 1065560.748 & 9.7824 \\
\hline 35 & $04 / 02 / 2040$ & 07:20:00 a.m. & 1171468.173 & 9.7997 \\
\hline 36 & $04 / 02 / 2040$ & 08:15:00 a.m. & 1103895.603 & 9.7886 \\
\hline 37 & $04 / 02 / 2040$ & 09:10:00 a.m. & 1051584.276 & 9.7802 \\
\hline 38 & $04 / 02 / 2040$ & 07:10:00 a.m. & 1152019.283 & 9.7965 \\
\hline 39 & $04 / 02 / 2040$ & 07:10:00 a.m. & 1177320.73 & 9.8007 \\
\hline
\end{tabular}




\begin{tabular}{|c|c|c|c|c|}
\hline 40 & 04/02/2040 & 08:35:00 a.m. & 1091006.86 & 9.7865 \\
\hline 41 & 04/02/2040 & 09:00:00 a.m. & 1080731.252 & 9.7849 \\
\hline 42 & $04 / 02 / 2040$ & 09:30:00 a.m. & 1043108.145 & 9.7788 \\
\hline 43 & $04 / 02 / 2040$ & 09:00:00 a.m. & 1068222.224 & 9.7829 \\
\hline 44 & $04 / 02 / 2040$ & 06:35:00 a.m. & 1211128.964 & 9.8064 \\
\hline 45 & $04 / 02 / 2040$ & 10:15:00 a.m. & 987720.5394 & 9.7702 \\
\hline 46 & $04 / 02 / 2040$ & 09:00:00 a.m. & 1073994.997 & 9.7838 \\
\hline 47 & $04 / 02 / 2040$ & 09:30:00 a.m. & 1030703.482 & 9.7769 \\
\hline 48 & 04/02/2040 & 08:25:00 a.m. & 1093219.438 & 9.7868 \\
\hline 49 & $04 / 02 / 2040$ & 09:00:00 a.m. & 1064352.991 & 9.7822 \\
\hline 50 & $04 / 02 / 2040$ & 08:50:00 a.m. & 1082698.251 & 9.7852 \\
\hline 51 & $04 / 02 / 2040$ & 07:55:00 a.m. & 1130934.916 & 9.7930 \\
\hline 52 & $04 / 02 / 2040$ & 08:15:00 a.m. & 1092074.665 & 9.7867 \\
\hline 53 & $04 / 02 / 2040$ & 09:30:00 a.m. & 1042291.335 & 9.7787 \\
\hline 54 & $04 / 02 / 2040$ & 07:05:00 a.m. & 1184596.754 & 9.8019 \\
\hline 55 & $04 / 02 / 2040$ & 08:05:00 a.m. & 1122029.178 & 9.7915 \\
\hline 56 & $04 / 02 / 2040$ & 07:55:00 a.m. & 1126187.987 & 9.7922 \\
\hline 57 & $04 / 02 / 2040$ & 07:50:00 a.m. & 1149371.189 & 9.7960 \\
\hline 58 & $04 / 02 / 2040$ & 07:55:00 a.m. & 1134265.283 & 9.7935 \\
\hline 59 & $04 / 02 / 2040$ & 09:00:00 a.m. & 1075692.02 & 9.7841 \\
\hline 60 & $04 / 02 / 2040$ & 09:10:00 a.m. & 1053956.134 & 9.7806 \\
\hline 61 & $04 / 02 / 2040$ & 09:00:00 a.m. & 1076784.002 & 9.7842 \\
\hline 62 & $04 / 02 / 2040$ & 09:50:00 a.m. & 1025087.569 & 9.7760 \\
\hline 63 & $04 / 02 / 2040$ & 08:05:00 a.m. & 1127461.508 & 9.7924 \\
\hline 64 & 04/02/2040 & 08:25:00 a.m. & 1093281.558 & 9.7869 \\
\hline 65 & $04 / 02 / 2040$ & 08:05:00 a.m. & 1102136.001 & 9.7883 \\
\hline 66 & $04 / 02 / 2040$ & 07:45:00 a.m. & 1131042.199 & 9.7931 \\
\hline 67 & $04 / 02 / 2040$ & 10:00:00 a.m. & 1005386.534 & 9.7729 \\
\hline 68 & 04/02/2040 & 09:30:00 a.m. & 1033617.049 & 9.7774 \\
\hline 69 & $04 / 02 / 2040$ & 09:20:00 a.m. & 1046259.296 & 9.7793 \\
\hline 70 & $04 / 02 / 2040$ & 07:55:00 a.m. & 1129240.62 & 9.7927 \\
\hline 71 & 04/02/2040 & 09:00:00 a.m. & 1060700.696 & 9.7817 \\
\hline
\end{tabular}




\begin{tabular}{|c|c|c|c|c|}
\hline 72 & $04 / 02 / 2040$ & 08:30:00 a.m. & 1090620.645 & 9.7865 \\
\hline 73 & $04 / 02 / 2040$ & 07:20:00 a.m. & 1168951.571 & 9.7993 \\
\hline 74 & $04 / 02 / 2040$ & 07:55:00 a.m. & 1119488.378 & 9.7911 \\
\hline 75 & $04 / 02 / 2040$ & 07:20:00 a.m. & 1161082.375 & 9.7980 \\
\hline 76 & $04 / 02 / 2040$ & 08:15:00 a.m. & 1093493.614 & 9.7869 \\
\hline 77 & $04 / 02 / 2040$ & 09:20:00 a.m. & 1032268.331 & 9.7772 \\
\hline 78 & $04 / 02 / 2040$ & 09:20:00 a.m. & 1039760.884 & 9.7783 \\
\hline 79 & $04 / 02 / 2040$ & 08:15:00 a.m. & 1113385.487 & 9.7901 \\
\hline 80 & $04 / 02 / 2040$ & 08:25:00 a.m. & 1105015.61 & 9.7887 \\
\hline 81 & $04 / 02 / 2040$ & 08:50:00 a.m. & 1067588.837 & 9.7828 \\
\hline 82 & $04 / 02 / 2040$ & 07:10:00 a.m. & 1153363.38 & 9.7968 \\
\hline 83 & $04 / 02 / 2040$ & 08:15:00 a.m. & 1117264.187 & 9.7907 \\
\hline 84 & $04 / 02 / 2040$ & 08:35:00 a.m. & 1099563.859 & 9.7879 \\
\hline 85 & $04 / 02 / 2040$ & 08:05:00 a.m. & 1113757.918 & 9.7902 \\
\hline 86 & $04 / 02 / 2040$ & 09:50:00 a.m. & 1027258.514 & 9.7763 \\
\hline 87 & $04 / 02 / 2040$ & 09:00:00 a.m. & 1083656.745 & 9.7853 \\
\hline 88 & $04 / 02 / 2040$ & 09:30:00 a.m. & 1029086.244 & 9.7767 \\
\hline 89 & $04 / 02 / 2040$ & 09:20:00 a.m. & 1039289.007 & 9.7782 \\
\hline 90 & $04 / 02 / 2040$ & 09:00:00 a.m. & 1070865.069 & 9.7833 \\
\hline 91 & $04 / 02 / 2040$ & 09:10:00 a.m. & 1039365.503 & 9.7783 \\
\hline 92 & $04 / 02 / 2040$ & 09:40:00 a.m. & 1011875.474 & 9.7740 \\
\hline 93 & $04 / 02 / 2040$ & 07:45:00 a.m. & 1137341.854 & 9.7941 \\
\hline 94 & $04 / 02 / 2040$ & 09:00:00 a.m. & 1069714.852 & 9.7831 \\
\hline 95 & $04 / 02 / 2040$ & 07:55:00 a.m. & 1111857.537 & 9.7899 \\
\hline 96 & $04 / 02 / 2040$ & 07:55:00 a.m. & 1105330.372 & 9.7889 \\
\hline 97 & $04 / 02 / 2040$ & 08:15:00 a.m. & 1101866.449 & 9.7883 \\
\hline 98 & $04 / 02 / 2040$ & 07:20:00 a.m. & 1167456.862 & 9.7990 \\
\hline 99 & $04 / 02 / 2040$ & 10:00:00 a.m. & 1006806.69 & 9.7731 \\
\hline 100 & $04 / 02 / 2040$ & 07:55:00 a.m. & 1134467.606 & 9.7935 \\
\hline \multirow[t]{2}{*}{ Promedio } & Fecha & Hora & $\mathrm{Km}$ & Vrel (Km/seg) \\
\hline & $04 / 02 / 2040$ & 08:37:24 a.m. & 1087412.671 & 9.7860 \\
\hline
\end{tabular}


Precisamente, al ser el escenario 45 el de mayor acercamiento, se aprovecha para hacer el análisis de la velocidad de escape. El máximo acercamiento se da a una distancia de $987,720.54 \mathrm{~km}$ y la velocidad relativa entre los cuerpos es de $9.7702 \mathrm{~km} / \mathrm{seg}$. A esta distancia lejana, de casi un millón de kilómetros, la velocidad de escape deberá ser relativamente baja (recordar que la velocidad de escape sobre la superficie de la Tierra es de $11.2 \mathrm{~km} / \mathrm{seg}$ ) y efectivamente, esta velocidad de escape es de sólo $0.8979 \mathrm{~km} / \mathrm{seg}$, mucho menor a la velocidad relativa entre ambos cuerpos. Por lo tanto, 2011AG5 no será atrapado por la gravedad terrestre.

Como dato adicional vale despejar de la ecuación de la velocidad de escape, la distancia a la cual, con una velocidad relativa de $9.7702 \mathrm{~km} / \mathrm{seg}$, el cuerpo podría ser atraído por la Tierra. Despejando de la ecuación se obtiene:

$$
r=\frac{2 G M}{V_{e}^{2}}
$$

Si la velocidad de escape se iguala a la velocidad relativa entre ambos cuerpos se obtiene una distancia de $8343.03 \mathrm{~km}$. A una distancia menor, la velocidad de escape será mayor a la velocidad relativa por lo que el cuerpo será atraído por la fuerza gravitacional de la Tierra. Nótese que la distancia es medida desde el centro de la Tierra, por lo que esta distancia correspondería a cerca de 2000 kilómetros sobre la superficie de nuestro planeta.

\section{CONCLUSIONES}

Se ha modelado el movimiento orbital del objeto NEA 2011AG5 para un período aproximado de 28 años, que abarca desde el año 2012 hasta el año 2040. Este seguimiento se ha dado en virtud de los cálculos iniciales que brindaban una probabilidad relativamente alta de impacto contra la Tierra para el año 2040. Los parámetros orbitales del 18 de abril de 2013 muestran la probabilidad de impacto en la práctica es cero (por la precisión del programa utilizado se sabe que este valor de probabilidad es mucho menor a 10-308). Este escenario muestra una distancia estimada de 1,087,412 $\mathrm{Km}$ con una desviación estándar de 46,637 Km, en un acercamiento probable calculado para el 4 de febrero de 2040 a las $8 \mathrm{~h} 37 \mathrm{~m}$, con una desviación estándar de 48m04s. La probabilidad de impacto se concluye nula.

Finalmente, a modo de confirmación, se ha estimado que la distancia a la cual tendría que pasar 2011AG5 para ser atraído por la fuerza gravitacional de la Tierra a una velocidad relativa de $9.77 \mathrm{~km} / \mathrm{seg}$, será de $8343.03 \mathrm{~km}$, o sea apenas cerca de $2000 \mathrm{~km}$ sobre la superficie terrestre. 


\section{BIBLIOGRAFÍA}

- Chodas, P. (2016). Near Earth Object Program. Obtenido de http://neo.jpl.nasa. gov/stats/

- Chodas, P. (2016). Near Earth Object Program. Obtenido de http://neo.jpl.nasa. gov/neo/groups.html

- Chodas, P. (2016). Near Earth Object Program. Obtenido de Impact Risk: http:// neo.jpl.nasa.gov/risk/removed.html

- Chodas, P. (2016). Sentry Risk Table. Obtenido de http://neo.jpl.nasa.gov/risk/

- Chodas, P. (2016). The Palermo Technical Impact Hazard Scale. Obtenido de http://neo.jpl.nasa.gov/risk/doc/palermo.html

- Fernández, J., Gallardo, T., \& Brunini, A. (2002). Icarus, 358.

- Lissauer, J. (2013). Fundamental Planetary Science. Cambridge University Press.

- Navarro, J. J., Catalá Poch, M. A., \& Núñez de Murga, J. (2007). Astronomía Esférica y Mecánica Celeste. Barcelona: Edicions de la Universitat de Barcelona.

- Park, R. (2015). JPL Small-Body Database Browser. Obtenido de http://ssd.jpl. nasa.gov/sbdb.cgi?sstr=2011\%20AG5\&orb=1

- Rubin, A. E., \& Grossman, J. N. (2010). Meteorite and meteoroid: New comprehensive definitions. Meteoritics \& Planetary Science, 114-122.

- Seeds, M. (2008). Foundations of Astronomy. Franklin and Marshall College. 